\title{
Sphingolipid de novo biosynthesis is essential for intestine cell survival and barrier function
}

\author{
Zhiqiang Li ${ }^{1,2}$, Inamul Kabir ${ }^{1}$, Gladys Tietelman' ${ }^{1}$ Chongmin Huan' ${ }^{1}$, Jianglin Fan ${ }^{3}$, Tilla Worgall ${ }^{4}$ and Xian-Cheng Jiang ${ }^{1,2}$
}

\begin{abstract}
Serine palmitoyltransferase (SPT) is the rate-limiting enzyme for sphingolipid biosynthesis. SPT has two major subunits, SPTLC1 and SPTLC2. We previously found that liver Sptlc2 deficiency in early life impairs the development of adherens junctions. Here, we investigated the role of Spt/c2 deficiency in intestine. We treated Spt/c2-Flox/villin-Cre-ER ${ }^{T 2}$ mice with tamoxifen (days 1, 2, and 3) to ablate Spt/c2 specifically in the intestine. At day 6 after tamoxifen treatment, Spt/c2deficient mice had significantly decreased body weight with concurrent diarrhea and rectal bleeding. The number of goblet cells was reduced in both large and small intestine of Spt/c2-deficient mice compared with controls. Spt/c2 deficiency suppressed the level of mucin2 in the colon and increased circulating lipopolysaccharides, suggesting that SPT activity has a housekeeping function in the intestine. All Spt/c2-deficient mice died 7-10 days after tamoxifen treatment. Notably, supplementation with antibiotics and dexamethasone reduced lethality by $70 \%$. We also found that colon specimens from patients with inflammatory bowel diseases had significantly reduced Spt/c2 expression, SPTLC2 staining, and goblet cell numbers. SPT activity is crucial for intestinal cell survival and barrier function.
\end{abstract}

\section{Introduction}

SPT is the first and rate-limiting enzyme involved in sphingolipid biosynthesis ${ }^{1}$. The mammalian SPT holoenzyme is primarily a heterodimer composed of two protein subunits, SPTLC1 $(53 \mathrm{kDa})$ and SPTLC2 $(63 \mathrm{kDa})$, which share $20 \%$ amino acid sequence identity ${ }^{2,3}$. However, studies indicate the existence of a third subunit, SPTLC3, which has $68 \%$ identity to SPTLC2 ${ }^{4}$. Additionally, two low-molecular-weight proteins, ssSPTa and ssSPTb, enhance the activity and confer distinct acyl-CoA substrate specificities to mammalian SPT, similar to the yeast Tsc3p subunit ${ }^{5}$. A relatively recent discovery indicated that yeast ORM (orosomucoid) 1/ORM2 proteins also

\footnotetext{
Correspondence: X-C. Jiang (XJiang@downstate.edu)

'Department of Cell Biology, State University of New York, Downstate Medical Center, Brooklyn, NY 11203, USA

${ }^{2}$ Molecular and Cellular Cardiology Program, VA New York Harbor Healthcare System, Brooklyn, NY 11209, USA

Full list of author information is available at the end of the article

Zhiqiang Li and Inamul Kabir contributed equally to this work

Edited by P Pinton
}

associate with and negatively regulate SPT activity ${ }^{6}$, thus adding another layer of complexity. Based on this new observation, a new term "SPOTS complex" (SPTLC1/2, ORM1/2, Tsc3, Sac1) was proposed ${ }^{6}$. These studies provide a starting point for investigating how protein and lipid synthesis is coordinated during cell membrane biogenesis.

Perturbations in SPT activity have been linked to diseases. Specific mutations identified in Sptlc1 or Sptlc2 cause a rare genetic disorder called hereditary sensory and autonomic neuropathy type $1^{7-9}$. The lack of Sptlc1 or Sptlc2 in mice causes embryonic lethality ${ }^{10}$. SPTLC1/ SPTLC2 binds the cell polarity factor Par3 and modulates monocyte chemotaxis ${ }^{11}$. Park et al. ${ }^{12}$ and we ${ }^{13}$ reported that treatment of Apoe knockout (KO) mice with myriocin, a highly selective inhibitor of SPT activity, decreases plasma sphingomyelin levels (via oral administration) and atherosclerosis (via intraperitoneal injection). However, myriocin often causes severe gastrointestinal side- 
effects $^{14}$, but the basis is unknown. We recently reported that liver-specific Sptlc2 deficiency in mice during early life impairs hepatocyte polarity through decreasing the levels of membrane factors that are involved in the formation of adherens junctions, thus promoting liver tumorigenesis ${ }^{15}$. We proposed an important role for SPT activity in establishing cell polarity and tissue integrity. As is the case for hepatocytes, enterocyte polarity is essential for intestinal functions. Among these functions, intestinal barrier function is the most important one.

Recent studies have clearly demonstrated the role of gut microbiota in health and chronic gastrointestinal disease ${ }^{16}$, but our knowledge of gut sphingolipid biosynthesis and barrier function remains incomplete. Emerging evidence suggests that sphingolipid metabolism contributes to the development of inflammatory bowel disease (IBD). Sakata et al. ${ }^{17}$ demonstrated that blocking the generation of ceramides with the Sphingomyelinase inhibitor hinders mouse colitis. Fischbeck et al. ${ }^{18}$ showed that increasing ceramides in the gut by supplying mice with dietary sphingomyelins, a precursor of ceramides, and aggravates mouse colitis. Wang et al. ${ }^{19}$ found that alkaline ceramidase 3 deficiency aggravates colitis and colitisassociated tumorigenesis. Notably, intestinal permeability is influenced by membrane sphingolipids ${ }^{20}$.

To further address the relationship between sphingolipid biosynthesis and gastrointestinal diseases, we created a mouse line in which Sptlc2 could be inducibly knocked out in the intestine to evaluate the impact of SPT activity on intestinal barrier function. We hypothesized that Sptlc2 deficiency in the intestine impairs cell polarity through reducing sphingolipid levels in the plasma membrane; the consequent change in gut permeability then allows previously immune-transparent microbes to become targeted by the host immune system. However, what we found was that the blockage of sphingolipid de novo synthesis has a dramatic impact on intestinal cell survival and barrier function.

\section{Results}

Preparation of inducible intestine-specific Sptlc2 KO mice

We prepared intestine-specific non-inducible Sptlc2 KO mice by crossing Villin-Cre transgenic mice with Sptlc2Flox mice. No homozygous Sptlc2 KO mice could be obtained after screening more than 100 offspring, so we chose an inducible approach (Supplementary Figure S1A).

We first prepared Sptlc2-Flox mice. We then crossed these mice with heterozygous Villin-Cre-ER ${ }^{\mathrm{T} 2}$ mice to obtain two lines of mice: Sptlc2-Flox (heterozygous)/Villin-Cre-ER ${ }^{\mathrm{T} 2}$ (heterozygous) and Sptlc2-Flox (heterozygous) mice. We then crossed these two lines of mice to obtain experimental and control mice: Sptlc2-Flox (homozygous)/Villin-Cre-ER ${ }^{\mathrm{T} 2}$ (heterozygous) and WT mice (Supplementary Figure S1B). The genetic background of all these mice was C57BL/6. To prepare intestine-specific Sptlc2-deficient mice, tamoxifen $(0.5 \mathrm{mg}$ per mouse), dissolved in corn oil, was injected intraperitoneally into the male mice on days 1,2 , and 3 .
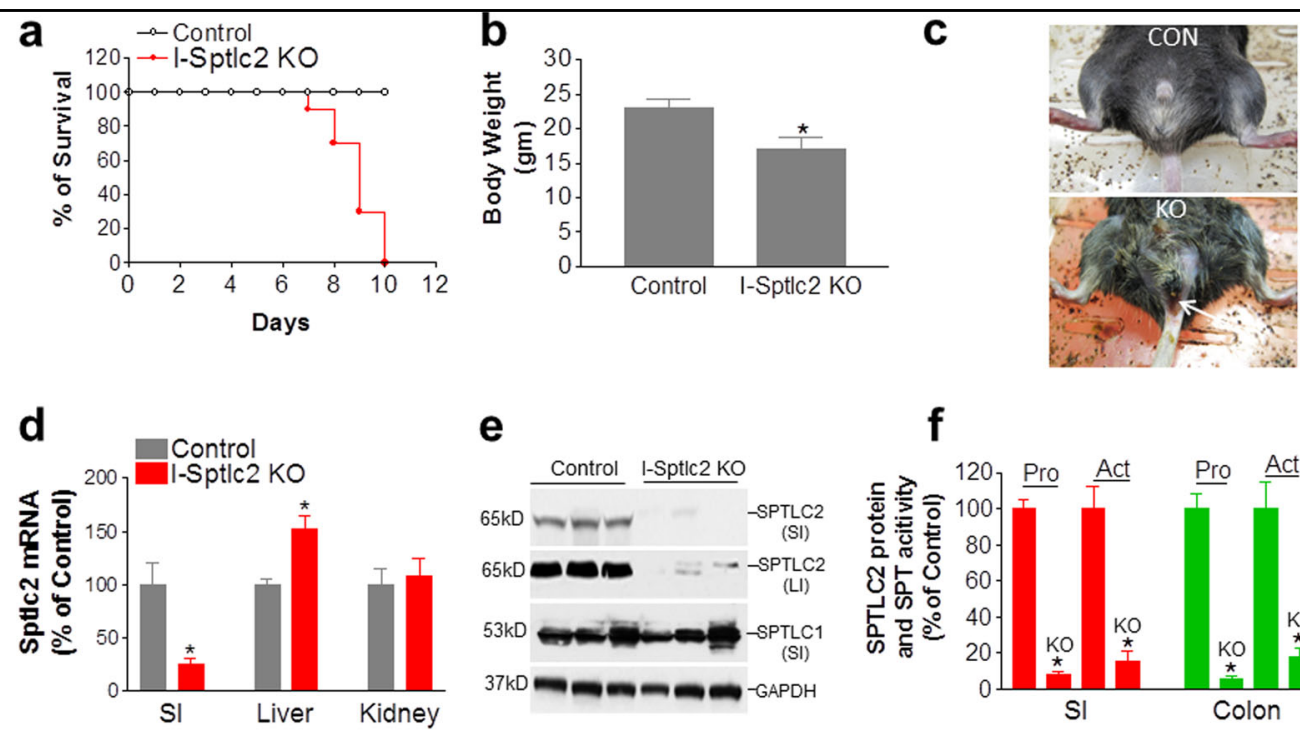

e

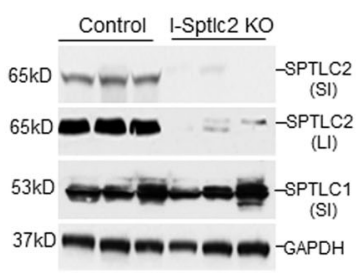

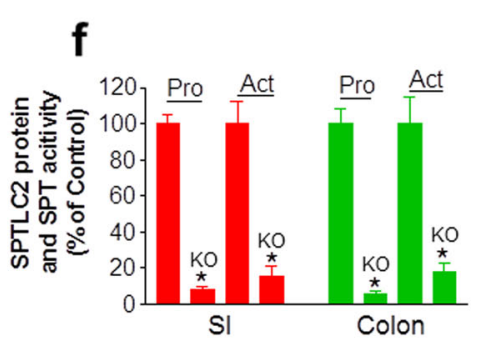

Fig. 1 Characterization of intestine-specific inducible Spt/c2 KO mice. Spt/c2-flox/Villin-Cre-ER ${ }^{\top 2}$ mice and controls were injected three times with tamoxifen ( $0.5 \mathrm{mg}$ per mouse, intraperitoneally). a Kaplan-Meier survival curve for intestine-specific Sptlc2-deficient mice, $n=20$. b Mouse body weight data on day 6 after tamoxifen treatment. c Image of a Sptlc2 mutant mouse with diarrhea and rectal bleeding. $\mathbf{d}$ SPTLC2 mRNA in different tissues was measured by real-time PCR on day 6 after tamoxifen treatment. e Western blotting for SPTLC2 and SPTLC1. $\mathbf{f}$ Quantification of SPTLC2 protein and activity in small intestine (SI) and large intestine (LI). I-Sptlc2 KO, intestine specific Spt/c2 KO mice. SI, small intestine. Values represent the mean $\pm S D, n=5,{ }^{*} P<0.01$ 
Tamoxifen-treated Sptlc2-Flox mice were used as controls. The tamoxifen-treated Sptlc2-Flox/Villin-CreER ${ }^{\mathrm{T} 2}$ mice died within 7-10 days after treatment, but this was not the case for the control mice (Sptlc2-Flox) (Fig. 1a). We thus characterized the mice on day 6 after tamoxifen treatment. The Sptlc2-deficient mice had reduced body weight (Fig. 1b, Supplementary Figure S2A) and food consumption (Supplementary Figure S2B), as well as diarrhea and rectal bleeding (Fig. 1c). The dying mice had significantly lower body temperature (Supplementary Figure S2C). SPTLC2 mRNA level was decreased by $80 \%$ in the intestine but not in other tissues (Fig. 1d). We then measured SPTLC2 protein level in homogenates of both small intestine and colon from Sptlc2-deficient mice and found that it was decreased by 95 and $92 \%$, respectively, compared with controls (Fig. 1e, f); SPT activity was also dramatically reduced in the small intestine (by $90 \%$ ) and colon (by 85\%) (Fig. 1f). Similar results were obtained with female mice (data not shown). Notably, there was no change in the overall level of SPTLC1 protein (Fig. 1e). We also measured mRNA levels of ORMDL1-3, ssSPTa, and ssSPTb in the colon. We found that all of them were significantly reduced (Supplementary Figure S3A and B).

\section{Intestine-specific Sptlc2 deficiency disrupts intestinal barrier function}

We measured sphingolipid levels in the plasma membrane of colon cells and found that Sptlc2-Flox/Villin$\mathrm{CreER}^{\mathrm{T} 2}$ mice had significantly smaller amounts of all tested ceramides and sphingomyelins (Table 1) after tamoxifen treatment, but no changes were observed for sphingosine-1-phosphate $(1.48 \pm 0.32$ vs. $1.17 \pm 0.10 \mathrm{ng} /$ mg protein). Necropsy revealed that the Sptlc2-deficient mice had a shorter colon compared with controls (Fig. 2a), a similar phenotype observed in inducible intestine-specific Kindlin 1 and $2 \mathrm{KO}$ mice $^{21}$.

We next assessed colon morphology via staining with hematoxylin and eosin (H\&E; Fig. 2b), periodic acid-Schiff (Fig. 2c), and Alcian Blue (Fig. 2d), which revealed that the ablation of Sptlc2 in intestine dramatically reduced the number of goblet cells in the colon compared with controls. We noticed that bacteria had infiltrated the mucosa of the Sptlc2-deficient mouse colon (Fig. 2e) and that the colon had disorganized crypts of Lieberkühn (Fig. 2b). Importantly, both TUNEL (terminal deoxynucleotidyl transferase-mediated dUTP nick end labeling) and immunostaining for cleaved caspase-3 clearly indicated that Sptlc2 deficiency aggravated apoptosis in the colon compared with the control group (Fig. 2f, g), and this was confirmed by western blotting for cleaved caspase-3 (Fig. 2h). We immunostained $\mathrm{T}$ and $\mathrm{B}$ lymphocytes with CD4 and B330 antibodies, respectively. We found both lymphocytes were significantly accumulated in Sptlc2 deficient colon (Supplementary Figure S4A and B).
We also found that significantly more $S p t l c 2$ deficient colon cells underwent proliferation (Fig. 3a, b) and differentiation, as suggested by western blotting for carbonic anhydrase $^{22}$ (Fig. 3d). The disorganization of the crypts of Lieberkühn was also confirmed by staining with DAPI (4',6-diamidine-2'-phenylindole dihydrochloride) (Fig. 3c).

We next visualized mouse colon with electron microscopy and found far fewer microvilli in the Sptlc2-deficient colon and that the microvilli were shorter and disorganized compared with controls (Fig. 3e, f), suggesting that inhibition of SPT activity in the intestine impaired cell integrity and structure.

Immunohistochemical analysis revealed that Sptlc2 ablation caused a dramatic reduction of E-cadherin, an important plasma-membrane protein for adherens junctions $^{23}$ (Fig. 4a). This was confirmed by western blotting for cadherin (Fig. 4b).

We next sought to measure mucin2 level in the Sptlc2deficient colon. Mucin2 is the major protein secreted by goblet cells, which provide a barrier function for the gut $^{24}$. We found that mucin 2 was reduced by $90 \%$ compared with control mice (Fig. 4c), suggesting a disruption of intestinal barrier function. We also found that the $\mathrm{KO}$ mice had a very high level of plasma lipopolysaccharides (LPS) compared with control mice (Fig. 4d). This high level of plasma LPS may have caused acute endotoxinemia, which would explain the lethal phenotype. We then measured the colon MAPK levels, which mediates inflammation. We found that Sptlc2 deficiency significantly increased the phosphorylation/activation of ERK and JNK but not p38 (Fig. 4e) in the colon compared with control mice. Moreover, we found that the $\mathrm{KO}$ mice have significantly higher IL- 6 and TNF $\alpha$ in the circulation (Fig. 4f).

We next evaluated the relationship between SPT activity and human inflammatory bowel diseases. We utilized a SPTLC2-specific antibody to immunostain colon samples that were obtained from human patients with Crohn's disease, chronic colitis, and ulcerative colitis, as well as control. Colons afflicted with the three disease types had much less intense SPTLC2 staining and fewer goblet cells and had greater lymphocyte infiltration compared with controls (Fig. 5a-c). Moreover, all diseased colons expressed less SPTLC2 mRNA, although this difference was not statistically significant for Crohn's disease (Fig. 5d). Thus, sphingolipid biosynthesis has a direct impact on human inflammatory bowel diseases.

We next sought to assess sphingolipid subspecies on the plasma membrane of enterocytes from the small intestine. After tamoxifen treatment, the Sptlc2-deficient mice had significantly decreased amounts of all tested ceramides and sphingomyelins (Table 1) but not sphingosine-1phosphate $(2.09 \pm 0.68$ vs. $1.69 \pm 0.21 \mathrm{ng} / \mathrm{mg}$ protein $)$. 


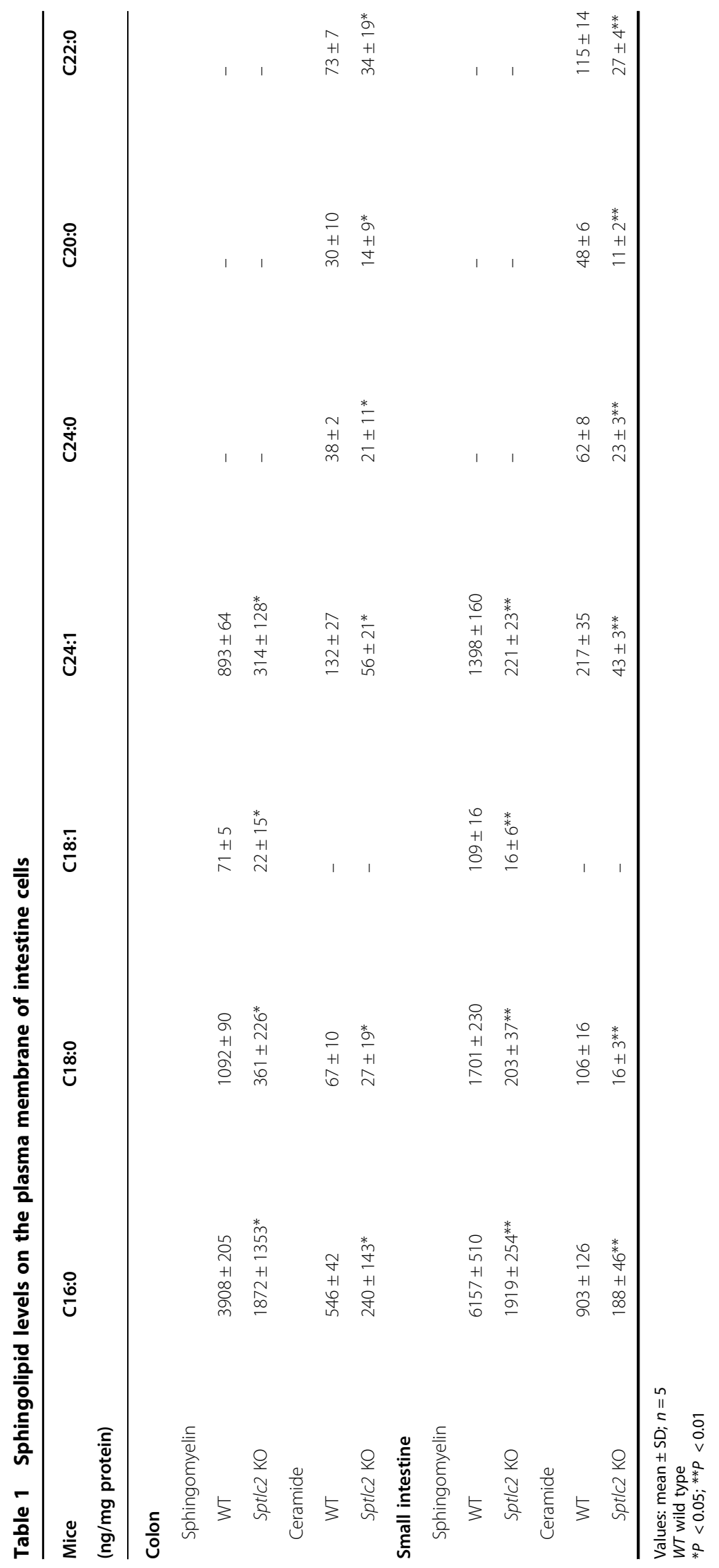




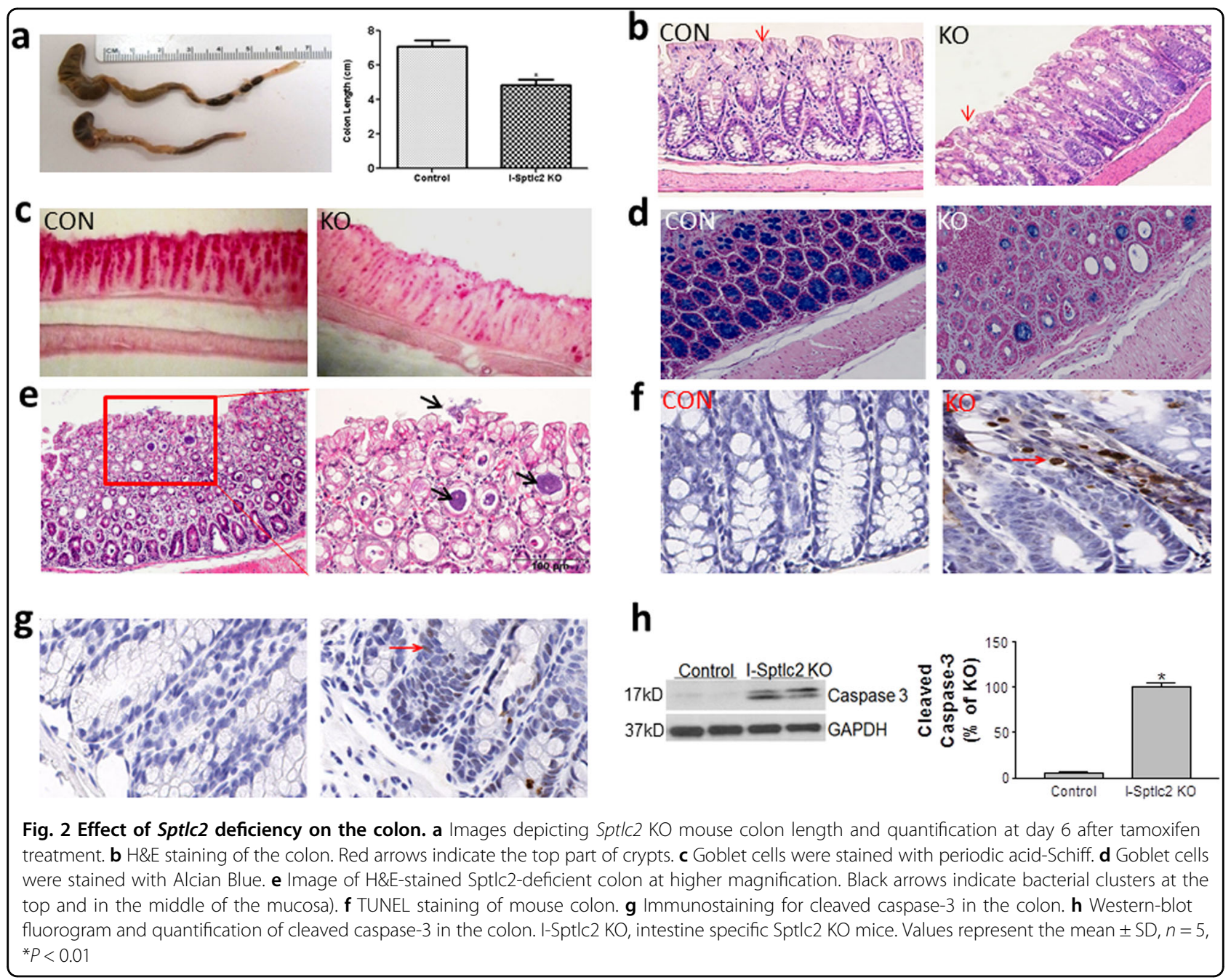

These changes could also have an impact small intestine morphology and pathology. Indeed, we found a significant reduction of both Paneth cells (Fig. 6a) and goblet cells (Fig. 6b) in the Sptlc2-deficient small intestine compared with the control. Next, we used western blotting to measure the levels of Niemann-Pick C1-like 1 (NPC1L1), CD36, ABCG8, and ABCA1, revealing a significant decrease in NPC1L1, CD36, and ABCA1 (62, 65, and 70, respectively; $P<0.01$ ); the level of ABCG8 was not significantly affected (Fig. 6c). We also used immunofluorescence staining to assess the level of apicalmembrane NPC1L1, which is involved in cholesterol uptake, and found that it was dramatically decreased in the small intestine of the Sptlc2 KO mice compared with the control (Fig. 6d). These results suggested that changes in the amounts of plasma-membrane sphingolipids can affect their localization on the apical membrane of enterocytes. Further, we found that the KO mice significantly reduced cholesterol absorption compared with controls (Supplementary Figure S5). Moreover, both
TUNEL and immunohistochemical analysis for cleaved caspase-3 clearly indicated that Sptlc2 deficiency also promoted apoptosis in the small intestine compared with the control group (Fig. 6e).

We also noticed that the Sptlc2-deficient mice had a significantly smaller spleen, fewer splenocytes (Supplementary Figure S6A), and lower levels of circulating white blood cells and lymphocytes (Supplementary Figure S6B). Moreover, compared with control mice, the Sptlc2-deficient mice exhibited substantially more inflammation in the lung, with more numerous infiltrated lymphocytes, and thicker septa (Supplementary Figure S6C), a typical phenotype of severe lung inflammation.

\section{Intestinal Spt/c2 deficiency-mediated lethality can be rescued}

The observed phenotypes suggested that the Sptlc2 deficiency-related lethality is related to the impairment of intestinal barrier function. Hence, we conjectured that, prior to tamoxifen administration, pre-treating the mice 
a

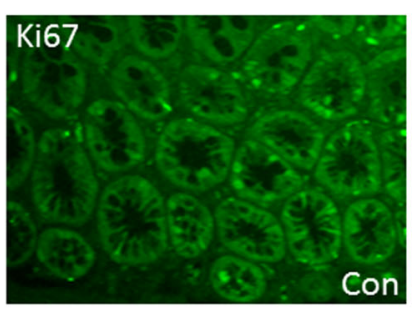

C

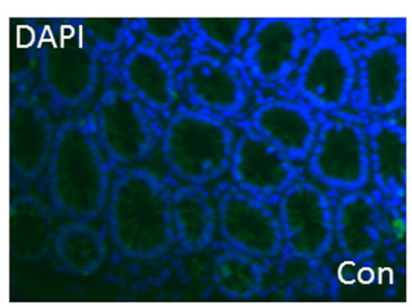

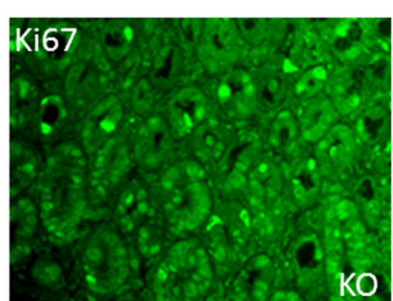

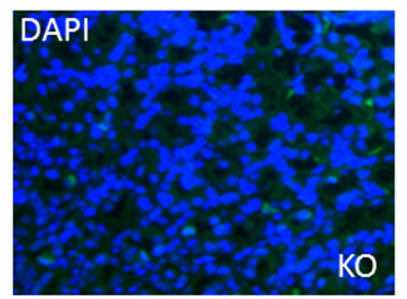

b
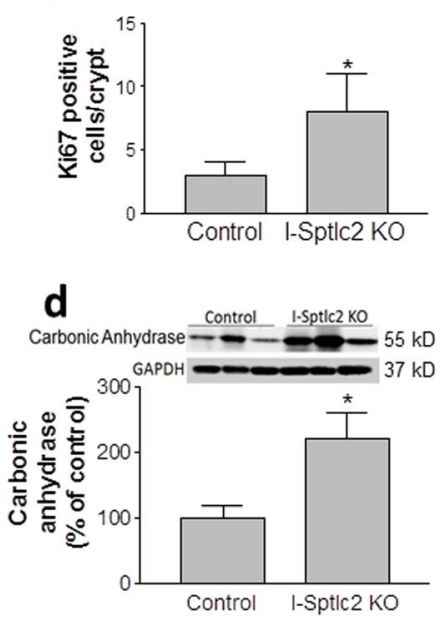

e

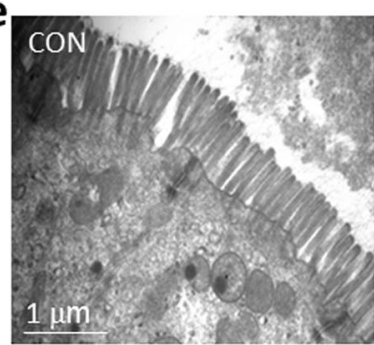

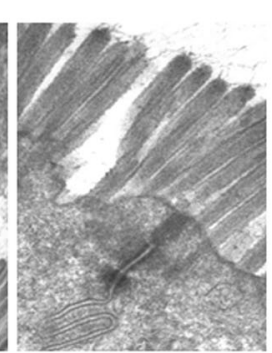

f

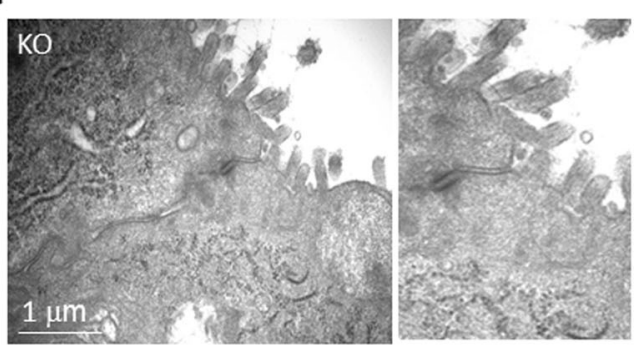

Fig. 3 Effect of Spt/c2 deficiency on colon cell differentiation and proliferation. a Immunostaining for Ki67. b Quantification of Ki67 in crypts. c DAPI staining to indicate nuclei. $\mathbf{d}$ Western-blot fluorogram and quantification of carbonic anhydrase in the colon. Values represent the mean \pm SD, $n$ $=5$, ${ }^{*} P<0.01$. e-f Electron microscopic image of mouse colon. All images are representative of five fields each. CON control, KO intestine-specific Sptlc2 knockout

with low absorbable antibiotics to cleanse the existing bacterial flora in the gut and with dexamethasone to induce an anti-inflammatory response could rescue the mice. Indeed, treatment of mice with antibiotics and dexamethasone rescued $70 \%$ of the mice (Fig. $7 \mathrm{a}$ ) and no death was observed after day 10, although the average body weight of the Sptlc2 KO mice was still less than that of the control at day 16 (Fig. 7b). We then measured SPTLC2 protein level after rescue (at day 16) and again found that the $\mathrm{KO}$ mice had dramatically decreased SPTLC2 in the colon (90\%; Fig. 7c).

We also measured plasma lipid levels in these rescued mice and found that these mice had significantly increased levels of plasma cholesterol $(55 \%, P<0.01)$, total phospholipids $(60 \%, P<0.01)$, and triglycerides $(40 \%, P<0.01)$ compared with controls (Fig. $7 \mathrm{~d})$. The female KO mice had a very similar phenotype (data not shown). Plasma apolipoprotein levels in Sptlc2 KO and control mice were assessed with reducing SDS-PAGE, which revealed a significant increase in plasma apoA-I $(P$ $<0.001)$ and apoE $(P<0.001)$ (Fig. $7 \mathrm{e})$. We also found a $70 \%$ reduction in apoB48 $(P<0.001)$, suggesting a defect in chylomicron production, and a small but significant $22 \%$ induction in apoB100 $(P<0.05)$ (Fig. 7e), suggesting enhanced production of very low-density lipoproteins in the liver.

We hypothesized that the observed lipid profile of Sptlc2 KO mice could be due to the effect of residual plasma LPS on the liver, because high plasma LPS correlates with hyperlipidemia ${ }^{25}$. Indeed, although treatment with antibiotics and dexamethasone rescued $70 \%$ of the Sptlc2 KO mice, the residual LPS in the circulation was still significantly higher than in controls $(3.2 \pm 0.8$ vs. 0.6 $\pm 0.1 \mathrm{EU} / \mathrm{ml}$; Fig. $7 \mathrm{f}$ ).

\section{Discussion}

In this study, we prepared intestine-specific inducible Sptlc2-deficient mice by crossing Sptlc2-Flox mice with Villin-Cre-ER ${ }^{\mathrm{T} 2}$ transgenic mice. We demonstrated that disruption of Sptlc2 in the intestine resulted in: (1) a significant decrease of ceramide and sphingomyelin in the plasma membrane of colon and small-intestine cells; (2) a promotion of intestinal cell apoptosis and death, and impairment of intestinal barrier function; and (3) 
a
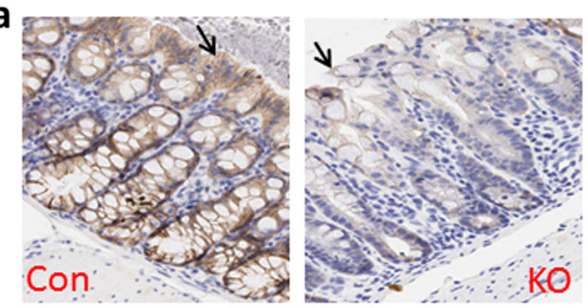

C

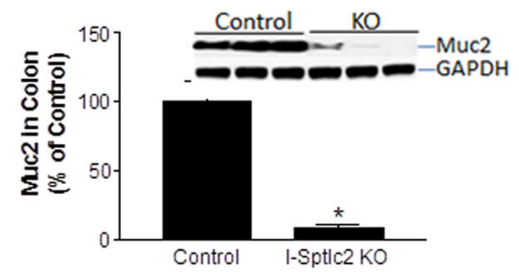

e

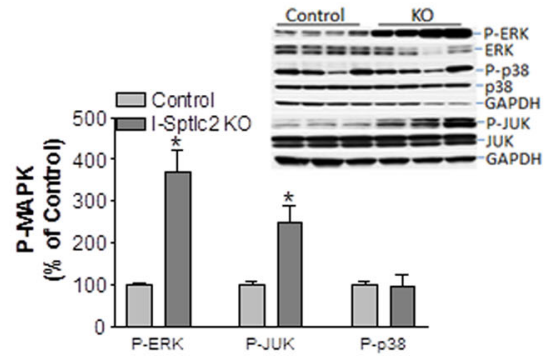

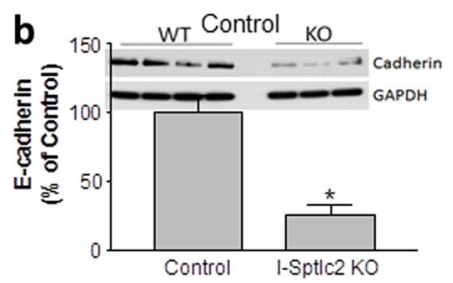

d

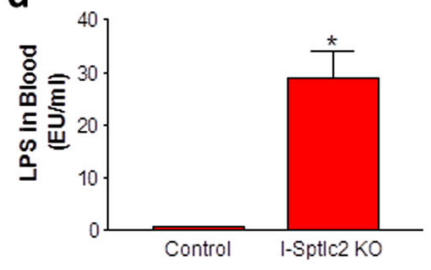

$\mathbf{f}$

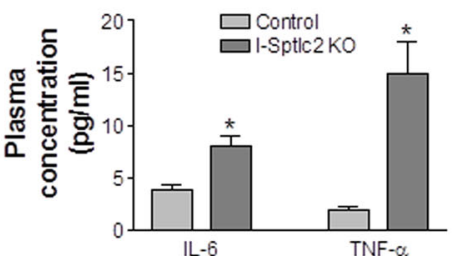

Fig. 4 Effect of Spt/c2 deficiency on cadherin, mucin2, and MAP kinases in the colon. a Immunostaining for E-cadherin in the colon. Black arrows indicate the top part of crypts. $\mathbf{b}$ Western-blot fluorogram and quantification of E-cadherin in the colon. $\mathbf{c}$ Western-blot fluorogram and quantification of mucin2. $\mathbf{d}$ LPS level in mouse plasma. e Western-blot fluorogram and quantification of phospho-ERK and total ERK, phospho-JNK and total JNK, and phospho-p38 and total p38 in the colon of Sptlc2-deficient and control mice. $\mathbf{f}$ Plasma IL-6 and TNF-a measurement. Values represent the mean $\pm S D, n=5,{ }^{*} P<0.01$. CON control, KO intestine-specific Sptlc2 knockout

accumulation of LPS in the circulation, presumably causing lethality. Moreover, pretreatment with antibiotics and dexamethasone could rescue intestine-specific Sptlc2 deficiency-mediated lethality. Importantly, intestinespecific Sptlc2-deficient mice can mimic certain phenotypes observed in human inflammatory bowel diseases, such as Crohn's disease, chronic colitis, and ulcerative colitis. To our knowledge, this is the first study linking sphingolipid de novo biosynthesis with intestine cell survival and barrier function.

Our key finding is that SPT activity is a critical factor for maintaining intestine integrity. It is most likely that the barrier defects observed in Sptlc2 KO mice are due to epithelial damage. Sptlc2 depletion in the intestine led to the death of all mice within 7-10 days after the administration of tamoxifen. Before death, all Sptlc2-deficient mice exhibited shivering, thus mimicking the phenotype of LPS-induced acute toxicity. These results suggest that Sptlc2 deficiency-mediated epithelium cell death impairs intestinal barrier integrity that is maintained by the mucus layer (secreted by goblet cells) and by antimicrobial agents (secreted by Paneth cells) ${ }^{26,27}$, thus promoting the infiltration of gut bacteria into the circulation.

The essential requirement for sphingolipid de novo biosynthesis in intestine cells consists with some other cell types. We found that liver Sptlc2 deficiency in early life impairs the development of hepatocyte polarity, resulting in severe jaundice and tumorigenesis ${ }^{15}$. Sphingolipid biosynthesis is also required for adipocyte survival, because adipocyte-specific Sptlc1 (another major subunit of SPT) KO mice exhibited a striking age-dependent loss of adipose tissues ${ }^{28}$ and adipocyte-specific Sptlc2 KO mice exhibited similar lipodystrophy phenotype ${ }^{29}$. The essential requirement for sphingolipid biosynthesis indicates that these cells are unable to acquire sufficient sphingolipids through alternative uptake and recycling pathways for cell survival.

The requirement for sphingolipid biosynthesis in intestine cells contrasts with some other cell types. We also found that an adult liver-specific Sptlc2 KO mouse exhibited reduced ceramide and sphingomyelin in both liver and plasma, but it displayed no apparent adverse effect on the hepatocytes ${ }^{30}$. In line with this, macrophage- 

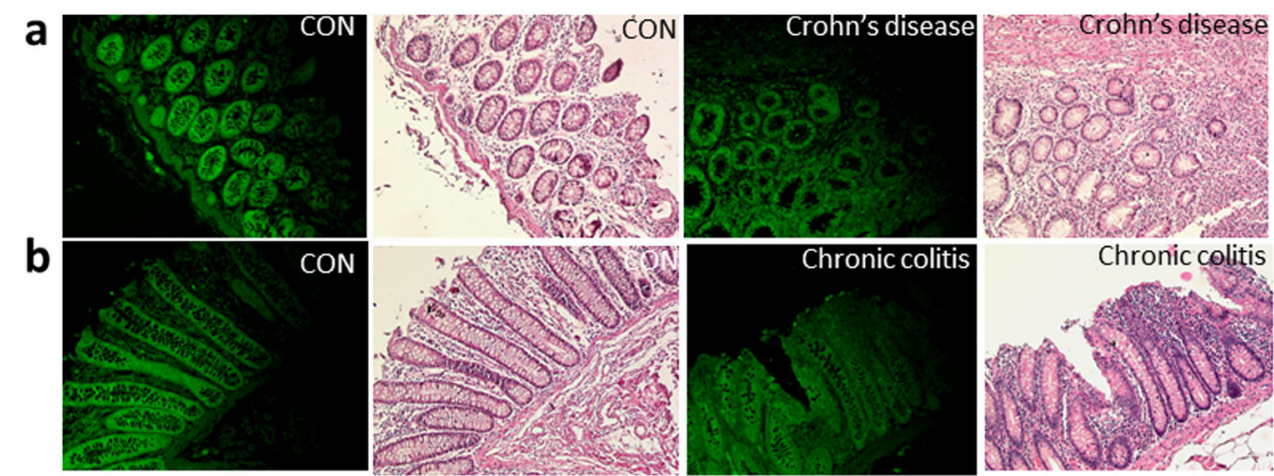

C
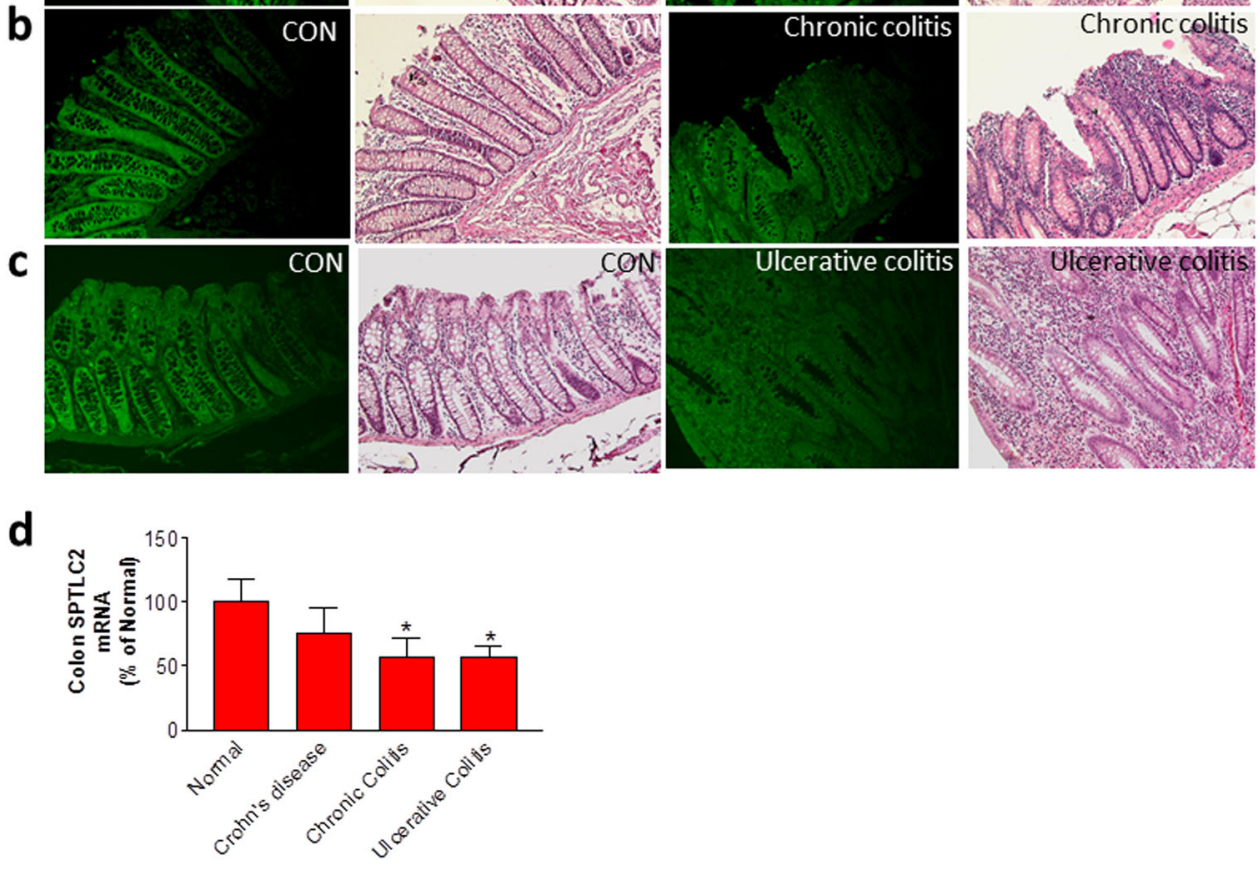

Fig. 5 Relationship between human inflammatory bowel diseases and SPTLC2. We utilized a SPTLC2-specfic antibody and H\&E stained colon samples from patients with Crohn's disease (a), chronic colitis (b), and ulcerative colitis (c), and compared the images with that of the control (CON). (d) We obtained human Crohn's and colitis tissue APCR array from QriGene Technologies, Inc. (Cat\# CCRT101), and then we did real-time PCR for SPTLC2 mRNA. Values represent the mean \pm SD, control group $n=4$, Crohn's disease group $n=6$, chronic colitis group $n=4$, ulcerative colitis group $n=14,{ }^{*} P<0.05$

specific Sptlc2 deficiency did not alter macrophage polarization and inflammatory capability ${ }^{31}$.

Which sphingolipid lacking, meditated by SPT deficiency, is responsible for the cell death? Sphingomyelin synthase 1 (Sms1) KO mice have been shown to have a severe lipodystrophy, with evidence of adipocyte cell death $^{32}$. It was inferred that ceramide accumulation, due to the block in conversion to sphingomyelin, was responsible for the phenotype. However, adipocytespecific Sptlc2 deficiency results in the reduction of sphingolipids, including both sphingomyelin and ceramide, yields a similar lipodystrophy phenotype as that of Sms1 deficiency ${ }^{28}$. We found in this study that both sphingomyelin and ceramide were decreased in intestine cells of our KO mice (Table 1). Thus, ceramide is not likely to play a role in cell death. The contribution of other sphingolipids to the phenotype was also considered. Sphingosine-1-phosphate, a sphingolipid that regulates processes such as inflammation ${ }^{33}$, could be another mediator. However, we did not observe a significant change of sphingosine-1-phosphate in the intestine.
Collectively, our results together with others suggest that the reduction of sphingomyelin may be a potential cause of this shared phenotype. We found that Sptlc2 deficiency reduced the levels of almost all sphingomyelins (Table 1), which are the major components of lipid rafts in the plasma membrane ${ }^{34}$. It is known that E-cadherin stabilization at cell-cell junctions requires raft microdomains ${ }^{35,36}$. Thus, Sptlc2 deficiency-mediated reduction in the amounts of sphingomyelins could decrease Ecadherin in lipid rafts and could result in losing cell polarity $^{15}$. Further studies are need for evaluating the relevance.

Inflammatory bowel diseases often involve environmental factors, infectious microbes, ethnicity, and a dysregulated immune system ${ }^{37}$. The genetic contribution is poorly understood ${ }^{37,38}$. Patients with an inflammatory bowel disease exhibit increased intestinal permeability, which favors bacterial/viral infections ${ }^{39}$. Consistent with this information, in most mouse models of colitis, recapitulation of the disease requires bacteria ${ }^{40}$. It is known that intestinal permeability is influenced by membrane 
a

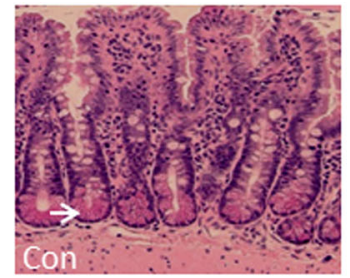

b
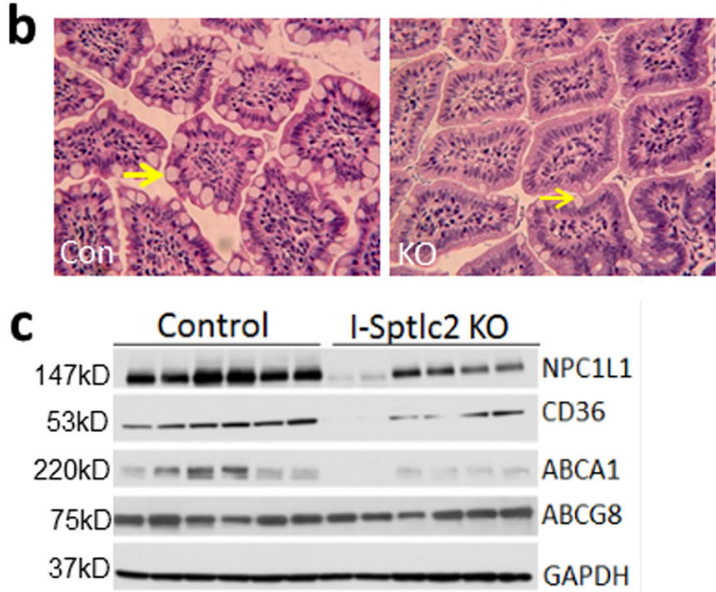

d
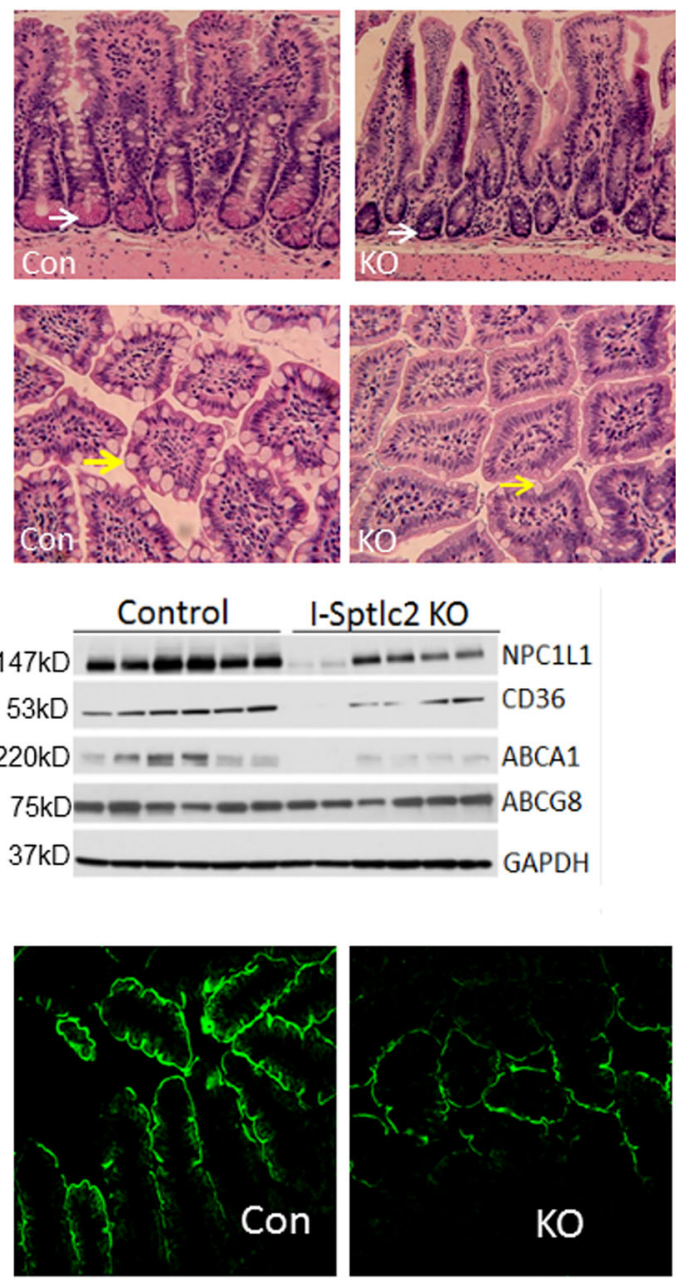
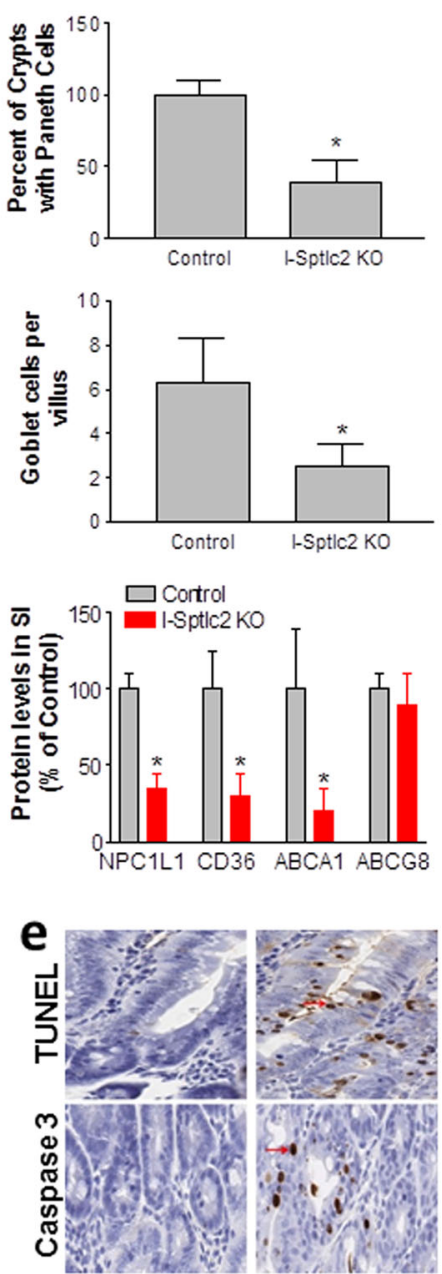

Fig. 6 Effect of Spt/c2 deficiency on the small intestine. a H\&E staining of Paneth cells and quantification in jejunum. White arrow indicates Paneth cells. $\mathbf{b} \mathrm{H} \& \mathrm{E}$ staining of and goblet cells and quantification in jejunum. Yellow arrow indicates goblet cells. Values represent the mean $\pm \mathrm{SD}, n=30,{ }^{*} P$ $<0.001$. c Western-blot fluorogram and quantification of NPC1L1, CD36, ABCA1, and ABCG8 in the plasma membrane of primary enterocytes. Values represent the mean $\pm \mathrm{SD}, n=5,{ }^{*} P<0.01$. $\mathbf{d}$ Immunostaining for NPC1L1 in the jejunum. e TUNEL and immunostaining for cleaved caspase-3 in the colon. Red arrows indicate apoptotic cells. All images are representative of five fields each

sphingolipids ${ }^{20}$. However, the mechanism by which permeability becomes compromised is still controversial. On the one hand, treatment fumonisin B1 (a fungal inhibitor of ceramide synthase that leads to depletion of ceramide as well as complex glycosphingolipids) treatment induces a primary defect in barrier function, thereby increasing intestinal epithelial permeability ${ }^{41}$. In line with this observation, treatment of pigs with fumonisin B1 was found to significantly increase intestinal colonization by pathogenic Escherichia coli ${ }^{42}$. Moreover, dietary glycosphingolipids can enhance intestinal permeability by enhancing cell junctions ${ }^{43}$. On the other hand, sphingomyelinase-mediated generation of ceramide might perturb barrier function as seen in inflammatory bowel diseases $^{44}$. The underlying cause(s) of this discrepancy between studies is unknown. It is possible that each of these studies was able to delineate only part of the overall mechanism. It is necessary to use an animal model to genetically deplete the pathway for sphingolipid de novo biosynthesis and then study the consequences. Therefore, in our present study, we created intestine-specific inducible Sptlc2 KO mice and found that a deficiency of sphingolipid de novo biosynthesis in the intestine promoted cell apoptosis, damaged intestine integrity, and increased intestinal permeability (Fig. 5f); moreover, patients with an inflammatory bowel disease were found to have a significantly lower level of colon SPTLC2 mRNA and of SPTLC2 staining intensity (Fig. 6a-d).

Necropsy revealed that the Sptlc2-deficient mice had a shorter colon compared with controls (Fig. 2a). How is this remodeling possible when the mice only have a lifespan of 6-10 days after tamoxifen induction? We also 
a

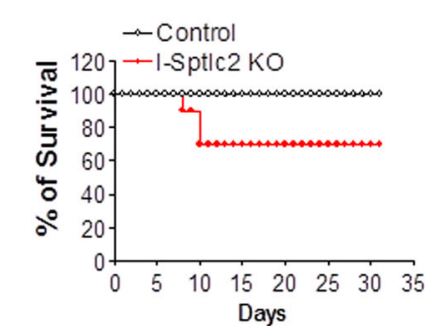

d

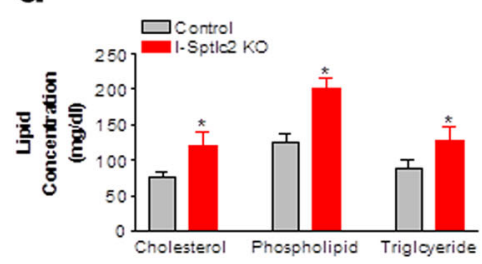

b
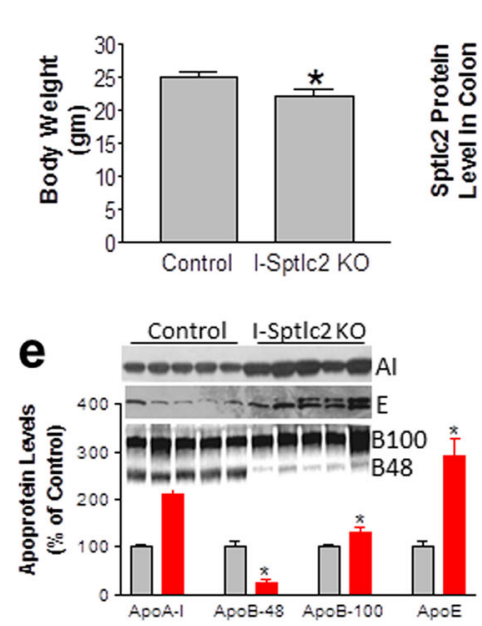
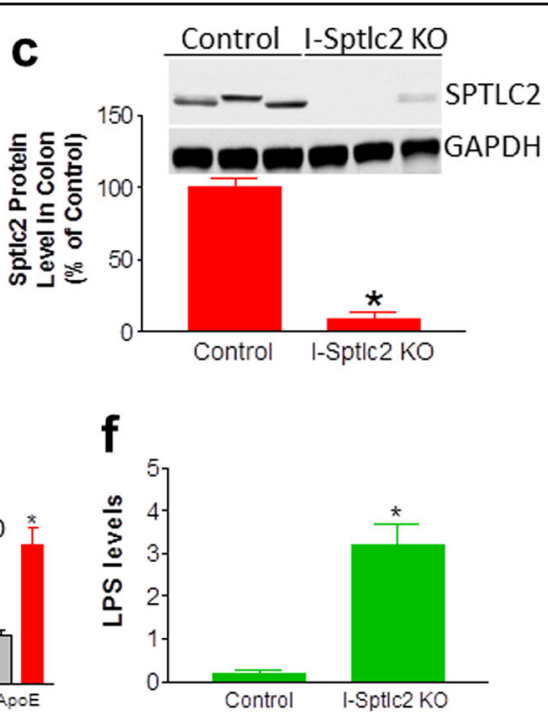

Fig. 7 Intestinal Spt/c2 deficiency-mediated lethality can be rescued. a Kaplan-Meier survival curve for intestine-specific Sptlc2-deficient mice after rescue with antibiotics and dexamethasone, $n=20$. b Body weight measurement at day 16. c Western-blot fluorogram and quantification of SPTLC2 in the colon at day 16. d Measurement of total plasma cholesterol, phospholipids, and triglycerides at day 16. e Western-blot fluorogram and quantification of plasma apolipoprotein levels at day 16. Plasma (0.2 $\mu$ l) was separated by gradient (4-15\% acrylamide) SDS-PAGE and subjected to western blotting with polyclonal antibodies against apoA-I, apoB, and apoE. $\mathbf{f}$ Plasma LPS levels at day 16 . Values represent the mean \pm SD, $n=5$, ${ }^{*}<<$ 0.05

puzzled by this observation. However, a most recent report also observed similar phenotype in three days after tamoxifen-induced villin-Cre-dependent intestinal deletion of Kindlin 1 and $2^{21}$. The authors observed that intestine specific deletion of kindlin 1 and 2 resulted in tight junction deletion and mucosal inflammation which was compatible with human ulcerative colitis ${ }^{21}$. We believe that shortened colon observed in our $\mathrm{KO}$ mice is related with intestinal cell death within 6-10 days after tamoxifen treatment.

It is well known that growth in epithelial cell proliferation and apoptosis correlates specifically to the inflammation activity of inflammatory bowel diseases ${ }^{45-47}$. In this study, we added one more evidence for this phenomenon. We found intestine Sptlc2 deficiency causes more intestine cell apoptosis (Fig. $2 \mathrm{f}-\mathrm{h}$ ) and more proliferations (Fig. 3a, b). We believe that Sptlc2 deficiency induces cell apoptosis which promotes the loss of gut epithelium. It might signal the stem cell proliferation and differentiation (Fig. 3d) in the crypt to replenish the dying enterocytes.

The mammalian SPT holoenzyme is primarily a heterodimer composed of two protein subunits, SPTLC1 and SPTLC2 ${ }^{2,3}$ or SPTLC1 and SPTLC ${ }^{4}$. SPTLC1 is not directly involved in the catalytic reaction; rather, it acts as an anchor that tethers SPTLC2 or SPTLC3 to the endoplasmic reticulum membrane ${ }^{48}$. The fact that SPTLC2 and SPTLC3, when expressed individually in Hek293 cells, increase SPT activity ${ }^{4}$ indicates that both subunits can act independently. However, SPTLC3 mRNA was undebatable in both Sptlc2 $\mathrm{KO}$ and control colons, although SPTLC1 and SPTLC2 could be easily detected (Supplementary Figure S7). The lethality of intestinespecific Sptlc2 deficiency clearly indicates that SPTLC2 is responsible for SPT activity in the intestine.

In conclusion, sphingolipid de novo synthesis is crucial for intestinal cell survival and barrier function. The blockage of SPT activity in the intestine causes intestinal cell death and impairment of barrier function.

\section{Materials and methods}

Generation of inducible intestine-specific Spt/c2 KO mice

Sptlc2-Flox mice were crossed with Villin-Cre-ER ${ }^{\mathrm{T} 2}$ (estrogen receptor T2), which yielded Sptlc2-Flox/VillinCre-ER ${ }^{\mathrm{T} 2}$ mice (Supplemental Fig. 1A). Mice were genotyped using PCR (Supplemental Fig. 1B). To delete Sptlc2 in the intestine, tamoxifen (Sigma Aldrich; $0.5 \mathrm{mg}$ per mouse, dissolved in $200 \mu \mathrm{l}$ of corn oil) was injected intraperitoneally every day for three times total. Controls were Sptlc2-Flox mice that had also been injected with tamoxifen. Both male and female mice (10 weeks old) with a C57BL/6J background were used. All experiments were approved by the Institutional Animal Care and Use Committee of the State University of New York Downstate Medical Center and conformed with the "Guide for the Care and Use of Laboratory Animals" published by the US National Institute of Health (NIH publication No. 8523, revised 1996). 
To rescue the lethality of Sptlc2 KO male and female mice, we treated the mice with low- absorption antibiotics $(25 \mathrm{mg} / \mathrm{kg}$ vancomycin; $20 \mathrm{mg} / \mathrm{kg}$ gentamycin; $5 \mathrm{mg} / \mathrm{kg}$ rifampicin) via oral gavage and with dexamethasone $(0.8$ mg per mouse, intramuscular) 2 weeks before tamoxifen treatment (see above) and continued for total 30 days. Thereafter, the mice were used for the assays described below.

\section{Isolation of plasma membrane from primary enterocytes}

Primary enterocytes were isolated according to previous reports ${ }^{49}$. Plasma membrane was isolated from primary enterocytes according to our published protocol ${ }^{50}$.

\section{Sphingolipid analyses}

Sphingomyelin, ceramide, and sphingosine-1-phosphate levels were measured in control and Sptlc2 KO enterocyte plasma membrane by liquid chromatography-coupled tandem mass spectrometry, as described ${ }^{34}$.

\section{FPLC}

Plasma lipid distribution was quantified by FPLC using a Superose 6B column. A $250-\mu \mathrm{l}$ aliquot of pooled plasma was injected onto the column and eluted with $50 \mathrm{mM}$ Tris- $\mathrm{HCl}(\mathrm{pH} 7.4)$ at a constant flow rate of $0.35 \mathrm{ml} / \mathrm{min}$. A $100-\mu \mathrm{l}$ aliquot from each fraction was used for the measurement of lipids.

\section{Gene expression analysis by real-time PCR}

Mice were sacrificed by cervical dislocation. The jejunum, liver, and kidneys were dissected and total RNA extracted from each tissue using the RNeasy Mini kit (Qiagen). cDNA was synthesized with an Invitrogen Superscript $^{\mathrm{TM}}$ III First-strand Synthesis kit. PCR was performed in a volume of $20 \mu \mathrm{l}$ with the SYBR Green kit from Applied Biosystems. The 18S rRNA was used as the internal control. The amplification program was: $95^{\circ} \mathrm{C}$ for $10 \mathrm{~min}$, followed by 40 amplification cycles of $95^{\circ} \mathrm{C}$ for 15 $\mathrm{s}$ and $60^{\circ} \mathrm{C}$ for $1 \mathrm{~min}$. Each sample was in triplicate. Relative gene expression is presented as mean $\pm \mathrm{SD}$. Mouse Sptlc2 primers were: forward, AGCCATTTCCGGTTTCGGAGA; reverse, TCCTCAAGTACCCGTTCCTCA. Mouse 18S rRNA primers were: forward, AGTCCCTGCCCTTTGTACACA; reverse, GATCCGAGGGCCTCACTAAAC.

\section{Western blotting}

Primary enterocyte homogenates were subjected to western blotting as described ${ }^{51}$ with antibodies against NPC1L1 (a gift from Dr. Bao Liang Song, Chinese Academy of Sciences), CD36 (Abcam), ATP-binding cassette transporter 8 (ABCG8; Novus Biologicals), and ABCA1 (Novus Biologicals). Homogenates of small intestine and colon tissue in PBS were used to determine levels of SPTLC2 (Proteintech Group) and SPTLC1 (BD Transduction). Colon tissue homogenate was used for western blotting for mucin2 (antibody from Novus Biologicals) and cleaved caspase-3 (antibody from Cell Signaling Technology). The activation of the MAPK pathway in colon was assessed using phospho-specific antibodies for ERK, p38, and JNK (Cell Signaling Technology, Cat. \#9910S). Total protein of ERK, p38, and JNK was also quantified in colon (Cell Signaling Technology, Cat. \#9926S). GAPDH was used as a loading control.

\section{SPT activity measurement}

Large intestine and small intestine from intestinespecific Sptlc2 KO and wild type mice were homogenized in SPT homogenization buffer (10 mM HEPES, $\mathrm{pH}$ 7.5, $10 \mathrm{mM}$ Sucrose, $1 \mathrm{mM}$ EDTA), and centrifugation at $4500 \mathrm{rpm}$ at $4{ }^{\circ} \mathrm{C}$ for $15 \mathrm{~min}$. The supernatant was adjusted to a final concentration of $0.25 \mathrm{M}$ sucrose, and then spin at 50,000 rpm (SW55) for $1 \mathrm{~h}$ at $4{ }^{\circ} \mathrm{C}$. The microsome pellet was resuspended in SPT microsome suspension buffer (10 mM HEPES, pH $7.5,0.25 \mathrm{M}$ Sucrose) for SPT activity assay.

SPT activity was assayed in $200 \mu \mathrm{l}$ of the reaction system containing $50 \mathrm{mM}$ HEPES, pH 7.5, $5 \mathrm{mM}$ EDTA, $5 \mathrm{mM}$ DTT, $50 \mu \mathrm{M}$ Pyridoxal phosphate, $200 \mu \mathrm{M}$ Palmitoyl$\mathrm{CoA}, 400 \mu \mathrm{M}$ Serine, and $0.5 \mu \mathrm{Ci}{ }^{14} \mathrm{C}$-serine. The reaction was initiated by the addition of microsomal protein, incubate at $37^{\circ} \mathrm{C}$ for $10 \mathrm{~min}$ in a water bath, and stopped by adding $400 \mu \mathrm{l}$ of $0.5 \mathrm{~N} \mathrm{NH} \mathrm{NH}_{4} \mathrm{OH}$. The lipids were extracted by adding $800 \mu \mathrm{l}$ of chloroform/methanol (2:1, $\mathrm{v} / \mathrm{v})$ and mixing vigorously. The organic phase was collected after centrifugation $(6000 \times g)$ for $10 \mathrm{~min}$ and dried under nitrogen gas. Lipids were dissolved in $20 \mu \mathrm{l}$ of chloroform and then were applied to TLC plate (chloroform/methanol/20\% ammonia, 14/6/1, v/v). The TLC plate was exposed to a phosphor screen and then scanned on a phosphor imager.

\section{H\&E staining}

The small intestine and colon were dissected and fixed overnight in $4 \%$ formalin. The tissue was embedded in paraffin and then sliced ( $5 \mu \mathrm{m}$ thick). Each slice was deparaffinized and stained with $H \& E$.

\section{Immunofluorescence staining and immunohistochemistry}

Intestinal tissues were fixed in $4 \%$ formalin overnight at $4{ }^{\circ} \mathrm{C}$ before preparation of 5 - $\mu \mathrm{m}$ paraffin sections. Prior to antibody staining, the sections were deparaffinized in xylene and rehydrated in a gradient series of ethanol, then subjected to high-temperature antigen retrieval in $50 \mathrm{mM}$ Tris- $\mathrm{HCl}$ (pH 9.0), $1 \mathrm{mM}$ EDTA. Sections were permeabilized and blocked in Tris- $\mathrm{HCl}$ with $0.5 \%$ Triton X-100 and $5 \%$ horse serum. The sections were incubated overnight at $4{ }^{\circ} \mathrm{C}$ with the primary antibodies rabbit anti- 
NPC1L1, goat anti-villin (Santa Cruz Biotechnology), and rabbit anti-Ki67 (Millipore). Slides were then incubated with fluorescently labeled secondary antibodies. Intestinal tissues fixed in $4 \%$ formalin were sent to Histowiz Inc. (SUNY Biotechnology Incubator) for immunohistochemical analysis of cleaved caspase- 3 and for TUNEL staining.

\section{Periodic acid-schiff staining and Alcian blue staining}

Deparaffinized and rehydrated intestinal tissue sections were treated with periodic acid (Abcam) for $5 \mathrm{~min}$ at room temperature. Slides were washed in distilled water and then stained with Schiff's reagent (Abcam) for $15 \mathrm{~min}$ at room temperature, followed by a 5-min wash in running tap water. The sections were then counterstained with hematoxylin, washed in running tap water for $2 \mathrm{~min}$, dehydrated twice in 95\% ethanol and twice in 100\% ethanol, and cover slipped. Proximal colon samples fixed in $4 \%$ formalin were sent to Histowiz Inc. for Alcian Blue staining.

\section{Measurement of LPS}

Plasma samples $(50 \mu \mathrm{l})$ were sent to Assaygate Inc. (Maryland) for bacterial LPS measurement.

\section{Information on human samples}

We obtained paraffin embedded human colon disease tissue array from US Biomax, Inc. (Cat\# BC05002a and CO809a). Colons from Crohn's disease, chronic colitis, ulcerative colitis, and controls were stained by SPTLC2 antibody and by H\&E. We obtained human Crohn's and colitis tissue qPCR array from QriGene Technologies, Inc. (Cat\# CCRT101), and then we did real-time PCR for SPTLC2 mRNA.

\section{Statistical analysis}

Data are expressed as mean \pm SD. Data between two groups were analyzed with the unpaired, two-tailed Student's $t$-test, and among multiple groups with analysis of variance followed by the Student-Newman-Keuls test. Statistical significance was set as $P<0.05$.

\section{Acknowledgements}

This work was partially supported by a Veterans Affairs Merit award 000900-01, National Institutes of Health grant R56HL121409. Author contributions: Z.L. and I.K. performed all the experiments, analyzed data; G.T. provided guidance for histology and pathology experiments; C.H. performed blood counts and FACS; J.F. performed histopathology experiments; T.W. performed LC/MS/MS for sphingomyelin and ceramide sub-species measurements; X.C.J. as the corresponding author, designed the entire project, interpreted the results, and wrote the manuscript.

\section{Author details}

'Department of Cell Biology, State University of New York, Downstate Medical Center, Brooklyn, NY 11203, USA. ²Molecular and Cellular Cardiology Program, VA New York Harbor Healthcare System, Brooklyn, NY 11209, USA.

${ }^{3}$ Department of Molecular Pathology, University of Yamanashi, Yamanashi,
Japan. ${ }^{4}$ Department of Medicine, Columbia University, New York, NY 10032 USA

\section{Conflict of interest}

The authors declare that they have no conflict of interest.

Supplementary Information accompanies this paper at https://doi.org/ 10.1038/s41419-017-0214-1.

Received: 13 September 2017 Revised: 13 November 2017 Accepted: 7 December 2017

Published online: 07 February 2018

\section{References}

1. Merrill, A. H. Jr. Characterization of serine palmitoyltransferase activity in Chinese hamster overy cells. Biochim. Et. Biophys. Acta 754, 284-291 (1983).

2. Weiss, B. \& Stoffel, W. Human and murine serine-palmitoyl-CoA transferasecloning, expression and characterization of the key enzyme in sphingolipid synthesis. Eur. J. Biochem. 249, 239-247 (1997).

3. Hanada, K., Hara, T. \& Nishijima, M. Purification of the serine palmitoyltransferase complex responsible for sphingoid base synthesis by using affinity peptide chromatography techniques. J. Biol. Chem. 275, 8409-8415 (2000).

4. Hornemann, T., Richard, S., Rutti, M. F., Wei, Y. \& von Eckardstein, A. Cloning and initial characterization of a new subunit for mammalian serinepalmitoyltransferase. J. Biol. Chem. 281, 37275-37281 (2006).

5. Han, G. et al. Identification of small subunits of mammalian serine palmitoyltransferase that confer distinct acyl-CoA substrate specificities. Proc. Natl Acad. Sci. USA 106, 8186-8191 (2009).

6. Breslow, D. K. et al. Orm family proteins mediate sphingolipid homeostasis. Nature 463, 1048-1053 (2010).

7. Bejaoui, K. et al. SPTLC1 is mutated in hereditary sensory neuropathy, type 1. Nat. Genet. 27, 261-262 (2001).

8. Gable, K. et al. Mutations in the yeast LCB1 and LCB2 genes, including those corresponding to the hereditary sensory neuropathy type I mutations, dominantly inactivate serine palmitoyltransferase. J. Biol. Chem. 277, 10194-10200 (2002).

9. Rotthier, A. et al. Mutations in the SPTLC2 subunit of serine palmitoyltransferase cause hereditary sensory and autonomic neuropathy type I. Am. J. Hum. Genet. 87, 513-522 (2010).

10. Hojjati, M. R., Li, Z. \& Jiang, X. C. Serine palmitoyl-CoA transferase (SPT) deficiency and sphingolipid levels in mice. Biochim. Biophys. Acta 1737, 44-51 (2005).

11. Tamehiro, N. et al. Cell polarity factor Par3 binds SPTLC1 and modulates monocyte serine palmitoyltransferase activity and chemotaxis. J. Biol. Chem. 284, 24881-24890 (2009).

12. Park, T. S. et al. Inhibition of sphingomyelin synthesis reduces atherogenesis in apolipoprotein E-knockout mice. Circulation 110, 3465-3471 (2004).

13. Hojjati, M. R. et al. Effect of myriocin on plasma sphingolipid metabolism and atherosclerosis in apoE-deficient mice. J. Biol. Chem. 280, 10284-10289 (2005).

14. Gullo, V. P., McAlpine, J., Lam, K. S., Baker, D. \& Petersen, F. Drug discovery from natural products. J. Ind. Microbiol. Biotechnol. 33, 523-531 (2006).

15. Li Z. et al. Liver serine palmitoyltransferase (SPT) activity deficiency in early life impairs adherens junctions and promotes tumorigenesis. Hepatology $\mathbf{6 4}$, 2089-2102 (2016)

16. Guinane, C. M. \& Cotter, P. D. Role of the gut microbiota in health and chronic gastrointestinal disease: understanding a hidden metabolic organ. Ther. Adv. Gastroenterol. 6, 295-308 (2013).

17. Sakata, A. et al. Acid sphingomyelinase inhibition suppresses lipopolysaccharide-mediated release of inflammatory cytokines from macrophages and protects against disease pathology in dextran sulphate sodiuminduced colitis in mice. Immunology 122, 54-64 (2007).

18. Fischbeck, A. et al. Sphingomyelin induces cathepsin D-mediated apoptosis in intestinal epithelial cells and increases inflammation in DSS colitis. Gut $\mathbf{6 0}$, 55-65 (2011).

19. Wang, K. et al. Alkaline ceramidase 3 deficiency aggravates colitis and colitisassociated tumorigenesis in mice by hyperactivating the innate immune system. Cell Death Dis. 7, e2124 (2016).

20. Abdel Hadi, L., Di Vito, C. \& Riboni, L. Fostering inflammatory bowel disease: sphingolipid strategies to join forces. Mediat. Inflamm. 2016, 3827684 (2016). 
21. Stremmel, W. et al. Genetic mouse models with intestinal-specific tight junction deletion resemble an ulcerative colitis phenotype. J Crohn's Colitis 11, 1247-1257 (2017).

22. Bekku, S. et al. Carbonic anhydrase I and II as a differentiation marker of human and rat colonic enterocytes. Res. Exp. Med. 198, 175-185 (1998).

23. Perez-Moreno, M. \& Fuchs, E. Catenins: keeping cells from getting their signals crossed. Dev. Cell 11, 601-612 (2006).

24. Zarepour, M. et al. The mucin Muc2 limits pathogen burdens and epithelial barrier dysfunction during Salmonella enterica serovar Typhimurium colitis. Infect. Immun. 81, 3672-3683 (2013).

25. Feingold, K. R. et al. Endotoxin rapidly induces changes in lipid metabolism that produce hypertriglyceridemia: low doses stimulate hepatic triglyceride production while high doses inhibit clearance. J. Lipid Res. 33, 1765-1776 (1992).

26. Pelaseyed, T. et al. The mucus and mucins of the goblet cells and enterocytes provide the first defense line of the gastrointestinal tract and interact with the immune system. Immunol. Rev. 260, 8-20 (2014).

27. Farin, H. F. et al. Paneth cell extrusion and release of antimicrobial products is directly controlled by immune cell-derived IFN-gamma. J. Exp. Med. 211, 1393-1405 (2014)

28. Alexaki, A. et al. De novo sphingolipid biosynthesis is required for adipocyte survival and metabolic homeostasis. J. Biol. Chem. 292, 3929-3939 (2017).

29. Lee, S. Y. et al. Adipocyte-specific deficiency of de novo sphingolipid biosynthesis leads to lipodystrophy and insulin resistance. Diabetes $\mathbf{6 6 , 2 5 9 6 - 2 6 0 9}$ (2017).

30. Li, Z. et al. Liver-specific deficiency of serine palmitoyltransferase subunit 2 decreases plasma sphingomyelin and increases apolipoprotein E levels. J. Biol. Chem. 284, 27010-27019 (2009).

31. Camell, C. D. et al. Macrophage-specific de novo synthesis of ceramide is dispensable for inflammasome-driven inflammation and insulin resistance in obesity. J. Biol. Chem. 290, 29402-29413 (2015).

32. Yano, M. et al. Increased oxidative stress impairs adipose tissue function in sphingomyelin synthase 1 null mice. PLoS One 8, e61380 (2013).

33. Pyne, N. J. \& Pyne, S. Sphingosine 1-phosphate and cancer. Nat. Rev. Cancer 10 489-503 (2010).

34. Chakraborty, M. et al. Myeloid cell-specific serine palmitoyltransferase subunit 2 haploinsufficiency reduces murine atherosclerosis. J. Clin. Invest. 123 1784-1797 (2013).

35. Causeret, M., Taulet, N., Comunale, F., Favard, C. \& Gauthier-Rouviere, C. Ncadherin association with lipid rafts regulates its dynamic assembly at cell-cell junctions in C2C12 myoblasts. Mol. Biol. Cell 16, 2168-2180 (2005).

36. Boscher, C. et al. Galectin-3 protein regulates mobility of N-cadherin and GM1 ganglioside at cell-cell junctions of mammary carcinoma cells. J. Biol. Chem. 287, 32940-32952 (2012).

37. Baumgart, D. C. \& Carding, S. R. Inflammatory bowel disease: cause and immunobiology. Lancet 369, 1627-1640 (2007).
38. Xavier, R. J. \& Podolsky, D. K. Unravelling the pathogenesis of inflammatory bowel disease. Nature 448, 427-434 (2007).

39. Lee, S. H. Intestinal permeability regulation by tight junction: implication on inflammatory bowel diseases. Intest. Res. 13, 11-18 (2015).

40. Elson, C. O. et al. Experimental models of inflammatory bowel disease revea innate, adaptive, and regulatory mechanisms of host dialogue with the microbiota. Immunol. Rev. 206, 260-276 (2005).

41. Enongene, E. N., Sharma, R. P., Bhandari, N., Voss, K. A. \& Riley, R. T. Disruption of sphingolipid metabolism in small intestines, liver and kidney of mice dosed subcutaneously with fumonisin B(1). Food Chem. Toxicol. 38, 793-799 (2000).

42. Bouhet, S., Le Dorze, E., Peres, S., Fairbrother, J. M. \& Oswald, I. P. Mycotoxin fumonisin B1 selectively down-regulates the basal IL-8 expression in pig intestine: in vivo and in vitro studies. Food Chem. Toxicol. 44, 1768-1773 (2006).

43. Park, E. J., Thomson, A. B. \& Clandinin, M. T. Protection of intestinal occludin tight junction protein by dietary gangliosides in lipopolysaccharide-induced acute inflammation. J. Pediatr. Gastroenterol. Nutr. 50, 321-328 (2010).

44. Bock, J., Liebisch, G., Schweimer, J., Schmitz, G. \& Rogler, G. Exogenous sphingomyelinase causes impaired intestinal epithelial barrier function. World J. Gastroenterol. 13, 5217-5225 (2007).

45. Sipos, F., Molnar, B., Zagoni, T., Berczi, L. \& Tulassay, Z. Growth in epithelial cell proliferation and apoptosis correlates specifically to the inflammation activity of inflammatory bowel diseases: ulcerative colitis shows specific p53- and EGFR expression alterations. Dis. Colon Rectum 48, 775-786 (2005).

46. Willing, B. P. \& Van Kessel, A. G. Enterocyte proliferation and apoptosis in the caudal small intestine is influenced by the composition of colonizing commensal bacteria in the neonatal gnotobiotic pig. J. Anim. Sci. 85, 3256-3266 (2007).

47. Moss, S. F., Attia, L., Scholes, J. V., Walters, J. R. \& Holt, P. R. Increased small intestinal apoptosis in coeliac disease. Gut 39, 811-817 (1996).

48. Yasuda, S., Nishijima, M. \& Hanada, K. Localization, topology, and function of the LCB1 subunit of serine palmitoyltransferase in mammalian cells. J. Biol. Chem. 278, 4176-4183 (2003).

49. Jiang, X. C. et al. Apolipoprotein B secretion and atherosclerosis are decreased in mice with phospholipid-transfer protein deficiency. Nat. Med. 7, 847-852 (2001).

50. Li, Z. et al. Reducing plasma membrane sphingomyelin increases insulin sensitivity. Mol. Cell. Biol. 31, 4205-4218 (2011).

51. Li, Z. et al. Deficiency in lysophosphatidylcholine acyltransferase 3 reduces plasma levels of lipids by reducing lipid absorption in mice. Gastroenterology 149, 1519-1529 (2015)

52. Gable, K., Slife, H., Bacikova, D., Monaghan, E. \& Dunn, T. M. Tsc3p is an 80amino acid protein associated with serine palmitoyltransferase and required for optimal enzyme activity. J. Biol. Chem. 275, 7597-7603 (2000). 